\title{
Cerebral Small Vessel Disease and Renal Function: Systematic Review and Meta-Analysis
}

\author{
Stephen D.J. Makin ${ }^{a}$ F.A.B. Cook ${ }^{b}$ Martin S. Dennis ${ }^{b}$ Joanna M. Wardlaw ${ }^{b}$ \\ ${ }^{a}$ Clinical Research Fellow, Neuroimaging Sciences, University of Edinburgh and b Division of Neuroimaging Sciences, \\ University of Edinburgh, Bramwell Dott Building, Western General Hospital, Edinburgh, UK
}

\section{Key Words}

Lacunar stroke $\cdot$ Renal function $\cdot$ Small vessel disease .

Proteinuria

\begin{abstract}
Background: The small vessel disease (SVD) that appears in the brain may be part of a multisystem disorder affecting other vascular beds such as the kidney and retina. Because renal failure is associated with both stroke and white matter hyperintensities we hypothesised that small vessel (lacunar) stroke would be more strongly associated with renal failure than cortical stroke. Therefore, we performed a systematic review and meta-analysis to establish first if lacunar stroke was associated with the renal function, and second, if cerebral small vessel disease seen on the MRI of patients without stroke was more common in patients with renal failure. Methods: We searched Medline and EMBASE for studies in adults with cerebral SVD (lacunar stroke or white matter hyper intensities (WMH) on Magnetic Resonance Imaging (MRI)), in which renal function was assessed (estimated glomerular filtration rate (eGFR) or proteinuria). We extracted data on SVD diagnosis, renal function, demographics and comorbidities. We performed two meta-analyses: first, we calculated the odds of renal impairment in lacunar (small vessel) ischaemic stroke compared to other ischaemic stroke subtypes (non-small vessel disease); and second, we calculated the odds of renal
\end{abstract}

impairment in non-stroke individuals with WMH on MRI compared to individuals without $\mathrm{WMH}$. We then performed a sensitivity analysis by excluding studies with certain characteristics and repeating the meta-analysis calculation. $\boldsymbol{R e}$ sults: After screening 11,001 potentially suitable titles, we included 37 papers reporting 32 studies of 20,379 subjects: 15 of stroke patients and 17 of SVD features in non-stroke patients. To diagnose lacunar stroke, 13/15 of the studies used risk factor-based classification (none used diffusionweighted MRI). 394/1,119 (35\%) of patients with lacunar stroke had renal impairment compared with 1,443/4,217 (34\%) of patients with non-lacunar stroke, OR $0.88,(95 \% \mathrm{CI}$ 0.6-1.30). In individuals without stroke the presence of SVD was associated with an increased risk of renal impairment (whether proteinuria or reduced eGFR) OR 2.33 (95\% Cl 1.803.01), when compared to those without SVD. After adjustment for age and hypertension, 15/21 studies still reported a significant association between renal impairment and SVD. Conclusion: We found no specific association between renal impairment and lacunar stroke, but we did find that in individuals who had not had a stroke, having more SVD features on imaging was associated with a worse renal function, which remained significant after controlling for hypertension. However, this finding does not exclude a powerful coassociate effect of age or vascular risk factor exposure. Future research should subtype lacunar stroke sensitively and control for major risk factors.

(c) 2014 S. Karger AG, Base

\begin{tabular}{ll}
\hline KARGER 125.s & $\begin{array}{l}\text { ( 2014 S. Karger AG, Basel } \\
\text { 1015-9770/14/0391-0039\$39.50/0 }\end{array}$ \\
$\begin{array}{l}\text { E-Mail karger@karger.com } \\
\text { www.karger.com/ced }\end{array}$ & $\begin{array}{l}\text { This is an Open Access article licensed under the terms of } \\
\text { the Creative Commons Attribution 3.0 Unported license } \\
\text { (CC BY 3.0) (www.karger.com/OA-license-WT), appli- } \\
\text { cable to the online version of the article only. }\end{array}$
\end{tabular}

J.M. Wardlaw, MD

Division of Neuroimaging Sciences, University of Edinburgh

Bramwell Dott Building, Western General Hospital, Crewe Rd

Edinburgh EH4 2XU (UK)

E-Mail joanna.wardlaw@ed.ac.uk 


\section{Introduction}

Impaired kidney function is associated with an increased risk of stroke [1], and reduced estimated glomerular filtration rate (eGFR) is associated with an increased risk of cerebral small vessel disease (SVD) such as white matter hyperintensities (WMH) [2], and lacunes. It has been proposed that SVD is a manifestation of an underlying multi-system endothelial disorder affecting the small vessels of the kidney, brain, heart, and retina [3], possibly mediated through inflammation $[4,5]$.

All studies of renal function and SVD are faced with the challenge of disentangling whether these disorders are a common consequence from shared risk factors or represent a causative relationship. One way of doing this is to compare patients with different stroke subtypes, that is, to compare patients with lacunar (small vessel) stroke to patients with other stroke subtypes, or to compare the prevalence of renal disease in individuals with imaging-determined SVD features. We hypothesised that: patients presenting with a symptomatic recent ischaemic lacunar stroke may have a greater risk of renal impairment than patients with the other ischaemic stroke subtypes that are associated with embolism or large vessel disease. Additionally, individuals without symptomatic stroke may be at increased risk of SVD on imaging if they have renal impairment, after risk factor adjustment.

We performed a systematic review of the literature to establish first the risk of renal impairment in patients with lacunar stroke compared to patients in other ischaemic stroke subtypes, and second, the risk of renal impairment in non-stroke participants with SVD features on imaging (e.g. WMH, lacunes) compared to those without SVD.

\section{Methods}

We followed the 'Preferred Reporting Items for Systematic Reviews and Meta-Analyses' (PRISMA) guidelines [6]. We searched MEDLINE (1966-present) and EMBASE (1981-present) using OVID (version OvidSP_UI03.08.01.105), using the search terms in the Supplementary Information - last search was conducted on April 2013. SM and FABC independently reviewed the titles to identify the relevant papers and extracted the data. We resolved the disagreements through mutual discussion and consultation with JMW. We hand-searched the past editions of the journal 'Stroke', and the abstracts of presentations at European Stroke Conferences from 2006-2013 (published in Cerebrovascular Diseases), and the reference lists of relevant review papers. We deemed a paper to be potentially relevant if it included a reference to the measurement of renal function or mentioned SVD in adult humans in the title, and then went on to read the whole paper.
We included studies that measured the renal function in living humans with either symptomatic lacunar stroke or imaging features of SVD. We included studies that described WMH, lacunes, or 'silent cerebral infarcts', as these terms are commonly used to refer to SVD on imaging $[7,8]$, but excluded atrophy as this was inconsistently reported. We excluded studies that were performed on animals or post mortem, that measured renal disease but did not investigate the renal function (e.g. studies of renal biopsy findings), and studies that only included participants with renal impairment.

We extracted data on study population; location (community or hospital); inclusion and exclusion criteria; diagnosis of stroke subtype; the details of any imaging and image analysis methods; the definition used of SVD; how renal function was measured and defined; blinding; the differences in risk factors between the participants with and without stroke/SVD; the numbers of participants with and without stroke/imaging features of SVD who had renal impairment; and any adjusted or unadjusted summary statistics such as Odds Ratios (OR). We contacted the authors if it was apparent that the data had been acquired but was not reported in the paper.

We considered renal impairment to be either a reduced eGFR $(<60 \mathrm{ml} / \mathrm{min}$, Stage $3 \mathrm{Chronic}$ Kidney Disease) or albuminuria either micro (30-300 ml/l) or macro (>300 ml/l). For the purpose of the meta-analysis, we used the definitions of renal impairment, lacunar stroke, and SVD from the individual studies within our overall definition.

We carried out two meta-analyses. First, we compared the risk of renal impairment in patients with lacunar stroke to patients in other ischaemic stroke sub-groups. Second, we compared the risk of renal impairment in non-stroke participants with SVD on imaging to those without SVD. The meta-analysis included all the studies in that had dichotomised participants into those with and without renal impairment. If a study gave the mean and standard deviation (SD) of the eGFR, we assumed a normal distribution and calculated the number of patients with an eGFR below 60 . We used a random effects model to account for differences in underlying study methodology. We performed analyses using Stats Direct (StatsDirect statistical software version 2.7.9 http://www.stats direct.com. England: StatsDirect Ltd., 2008) and RevMan (Version 5, Cochrane Collaboration).

Each meta-analysis followed the same procedure. We calculated the summary OR of all studies using the Mantel-Haenszel random effects model and assessed heterogeneity using the $\mathrm{I}^{2}$ statistic. We used a funnel plot to examine for publication bias. To assess the causes of heterogeneity and the risk of bias in individual studies we performed a sensitivity analysis by excluding certain studies with various characteristics and repeating the meta-analysis. Lastly, we examined the summary statistics of the individual studies that had carried out a multivariable analysis accounting for age and hypertension. No protocol was published externally.

\section{Results}

We identified 11,001 potentially suitable titles (fig. 1). Of these, we excluded 1,0676 titles because they did not refer to either renal function, or SVD in adults, and read 


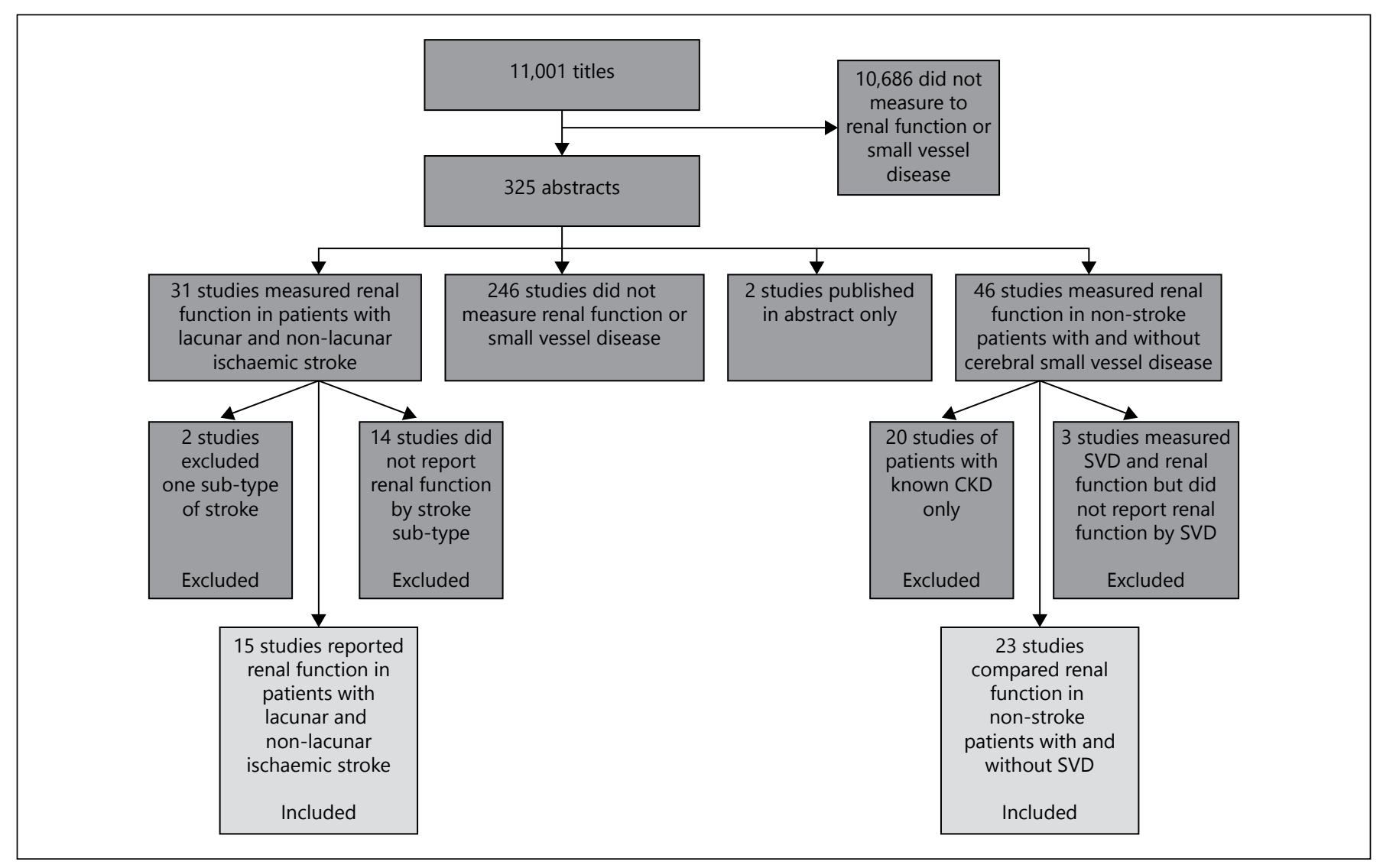

Fig. 1. Flow chart of search results.

the abstract or full paper of the remaining 325 references. We excluded 246 studies because they did not measure both renal function and SVD, two because they were published only in abstract, 20 because they only included patients with established kidney disease, 14 because they did not report the renal function of patients by stroke subtype and a further two because they only included one particular stroke sub-type. Full details of excluded studies are available on request.

We included 38 papers describing 32 studies of 20,379 participants: 15 studies [9-23] (45 of studies, $37 \%$ of patients) of stroke patients, and 17 studies [24-42] of healthy volunteers or non-stroke patients who had SVD defined on imaging alone (55 of studies, $63 \%$ of participants).

\section{Critical Appraisal of 15 Studies of Stroke Patients}

Study characteristics are summarised in tables 1 and 2 . Thirteen studies $[9,10,13-19,21-23,43]$ (87 of studies of stroke patients, $88 \%$ of stroke patients) recorded stroke subtype (lacunar or non-lacunar), while three recorded renal function in stroke patients with and without $\mathrm{WMH}$ on Magnetic Resonance Imaging (MRI) [9, 11, 12].

Most studies were from developed countries and varied with respect to inpatient and outpatient recruitment. One study [20] $(\mathrm{n}=958)$ included only young patients with a stroke but the rest included all ages (overall the mean age was 67). All studies measured the renal function as soon as possible after stroke, with the exception of one study of 96 patients which assessed renal function 6-8 weeks post-stroke [14].

Characteristics of the 13 Studies that Subtyped Stroke

Of the 13 studies that subtyped the stroke, only one [16] used the Oxfordshire Community Stroke Project (OCSP) [44] classification (8 of studies, 7\% of sub-typed stroke patients); all other studies used the risk-factor based Trial of Org 10,172 in Acute Stroke Treatment (TOAST) classification [45]. No studies used diffusion weighted MRI (DWI-MRI) in the acute phase. Four studies measured proteinuria $[13,14,19,22]$ (31 of studies, $9 \%$ of sub-typed stroke patients), and nine $[9,15-18,20-$ 
Table 1. Characteristics of studies included in the systematic review

\begin{tabular}{|c|c|c|c|c|c|c|}
\hline \multicolumn{2}{|c|}{ Study and population } & $\begin{array}{l}\text { Lacunar } \\
\text { stroke } \\
\text { definition }\end{array}$ & Imaging & $\begin{array}{l}\text { Definition } \\
\text { of renal } \\
\text { impairment }\end{array}$ & $\begin{array}{l}\text { Lacunar } \\
\text { ischaemic } \\
\text { stroke } \\
\text { impaired/total }\end{array}$ & $\begin{array}{l}\text { Non-lacunar } \\
\text { ischaemic } \\
\text { stroke } \\
\text { impaired/total }\end{array}$ \\
\hline \multicolumn{7}{|c|}{ Studies which compared renal function between lacunar and non-lacunar stroke } \\
\hline $\begin{array}{l}\text { Beamer } \\
{[14], 1999} \\
\text { USA }\end{array}$ & $\begin{array}{l}\text { Setting: } 2 \text { hospitals } \\
\text { Included: } 96 \text { patients up to } 7 \text { days post-stroke } \\
\text { Excluded: UTI and dialysis }\end{array}$ & TOAST & not clear & $\begin{array}{l}\text { proteinuria } \\
>20 \mathrm{mg} / \mathrm{l}\end{array}$ & $17 / 54$ & $11 / 42$ \\
\hline $\begin{array}{l}\text { Das [10], } \\
2012 \\
\text { Bangladesh }\end{array}$ & $\begin{array}{l}\text { Setting: neurology department } \\
\text { Included: } 60 \text { patients up to } 4 \text { weeks post-stroke } \\
\text { Excluded: known CKD }\end{array}$ & TOAST & $\mathrm{CT} / \mathrm{MRI}$ & $\begin{array}{l}\text { proteinuria } \\
20-200 \mathrm{ml} / 1\end{array}$ & $13 / 42$ & $6 / 18$ \\
\hline $\begin{array}{l}\text { MacWalter } \\
{[16], 2002} \\
\text { UK }\end{array}$ & $\begin{array}{l}\text { Setting: teaching hospital } \\
\text { Included: } 488 \text { patients } 48 \text { h post stroke } \\
\text { Excluded: dialysis }\end{array}$ & OCSP & $\mathrm{CT}$ & eGFR $<66$ & $121 / 134$ & $302 / 354$ \\
\hline $\begin{array}{l}\text { Rodríguez- } \\
\text { Yáñez [13], } \\
2006 \\
\text { Spain }\end{array}$ & $\begin{array}{l}\text { Setting: teaching hospital } \\
\text { Included: } 200 \text { patients within } 24 \mathrm{~h} \\
\text { Excluded: TPA/trial drug, brainstem stroke or known renal } \\
\text { disease }\end{array}$ & TOAST & $\mathrm{CT}$ & $\begin{array}{l}\text { proteinuria } \\
<30 \mathrm{mg} / \mathrm{l}\end{array}$ & $4 / 33$ & $45 / 167$ \\
\hline $\begin{array}{l}\text { Tsagalis } \\
\text { [19], } 2009 \\
\text { Greece }\end{array}$ & $\begin{array}{l}\text { Setting: teaching hospital stroke data bank } \\
\text { Included: } 2,155 \text { patients }<48 \text { h post stroke with } 2 \times \\
\text { creatinine measurements } \\
\text { Excluded: previous stroke }\end{array}$ & TOAST & $\mathrm{CT}$ & $\begin{array}{l}>50 \% \text { increase } \\
\text { in creatinine } \\
\text { from baseline }\end{array}$ & $72 / 378$ & $403 / 1454$ \\
\hline $\begin{array}{l}\text { Naganuma } \\
{[21], 2011} \\
\text { Japan }\end{array}$ & $\begin{array}{l}\text { Setting: registry of thrombolysis patients in } 10 \text { stroke units } \\
\text { Included: } 578 \text { patients who were thrombolysed for } \\
\text { ischaemic stroke } \\
\text { Excluded: patients disabled prior to stroke }\end{array}$ & TOAST & CT/MRI & eGFR $<60$ & $5 / 28$ & $181 / 550$ \\
\hline $\begin{array}{l}\text { Mostofsky } \\
{[18], 2009} \\
\text { USA }\end{array}$ & $\begin{array}{l}\text { Setting: emergency department } \\
\text { Included: } 1,175 \text { consecutive patients } \\
\text { Excluded: IN-hospital stroke }\end{array}$ & TOAST & CT/MRI & eGFR <60 & $105 / 339$ & $286 / 836$ \\
\hline $\begin{array}{l}\text { Ueda }[9] \\
2011 \\
\text { Japan }\end{array}$ & $\begin{array}{l}\text { Setting: stroke unit } \\
\text { Included: } 202 \text { consecutive ischaemic stroke patients } \\
\text { Excluded: acute kidney injury }\end{array}$ & TOAST & MRI & eGFR $<60$ & $12 / 38$ & $44 / 164$ \\
\hline $\begin{array}{l}\text { Putaala } \\
\text { [20], } 2011 \\
\text { Finland }\end{array}$ & $\begin{array}{l}\text { Setting: Helsinki young stroke registry } \\
\text { Included: } 958 \text { first stroke patients age } 15-19 \\
\text { Excluded: incomplete data or creatinine measured } 30 \text { days } \\
\text { post-stroke }\end{array}$ & TOAST & not clear & eGFR <60 & $17 / 130$ & $26 / 828$ \\
\hline $\begin{array}{l}\text { Hoshino } \\
{[17], 2012} \\
\text { Japan }\end{array}$ & $\begin{array}{l}\text { Setting: Neurology Department } \\
\text { Included: } 475 \text { stroke patients } \\
\text { Excluded: severe renal dysfunction, pre stroke disability }\end{array}$ & TOAST & $\mathrm{CT}$ & eGFR <60 & $29 / 92$ & $88 / 235$ \\
\hline $\begin{array}{l}\text { Kudo }[22] \\
2012 \\
\text { Japan }\end{array}$ & $\begin{array}{l}\text { Setting: single hospital } \\
\text { Included: } 525 \text { stroke patients } \\
\text { Excluded: missing data }\end{array}$ & TOAST & CT/MRI & $\begin{array}{l}\text { eGFR }<60 \text { and } / \\
\text { proteinuria }\end{array}$ & $\mathrm{r} 24 / 60$ & $168 / 264$ \\
\hline $\begin{array}{l}\text { Tsukamoto } \\
{[23], 2012} \\
\text { Japan }\end{array}$ & $\begin{array}{l}\text { Setting: neurology department } \\
\text { Included: } 639 \text { consecutive stroke patients } \\
\text { Excluded: dialysis patients }\end{array}$ & TOAST & CT/MRI & eGFR $<60$ & $32 / 104$ & $207 / 535$ \\
\hline $\begin{array}{l}\text { Chinda } \\
\text { [15], } 2012 \\
\text { Japan }\end{array}$ & $\begin{array}{l}\text { Setting: single hospital } \\
\text { Included: } 451 \text { consecutive stroke patients } \\
\text { Excluded: presented later than } 7 \text { days after stroke }\end{array}$ & TOAST & CT/MRI & eGFR <60 & $15 / 65$ & $79 / 224$ \\
\hline Studies whic & h compared renal function in stroke patients with WMH to th & se without & WMH & & & \\
\hline $\begin{array}{l}\text { Oksala } \\
{[11], 2010} \\
\text { Finland }\end{array}$ & $\begin{array}{l}\text { Setting: single hospital } \\
\text { Included: } 378 \text { consecutive ischaemic stroke patients aged } \\
55-85 \\
\text { Excluded: patients who were not Finnish or not living in } \\
\text { Helsinki }\end{array}$ & NA & $\begin{array}{l}1.0 \mathrm{~T} \\
\mathrm{MRI}\end{array}$ & eGFR $<60$ & $96 / 203$ & $56 / 175$ \\
\hline
\end{tabular}


Table 1. (continued)

\begin{tabular}{|c|c|c|c|c|c|c|}
\hline \multicolumn{2}{|c|}{ Study and population } & \multirow{2}{*}{$\begin{array}{l}\begin{array}{l}\text { Lacunar } \\
\text { stroke } \\
\text { definition }\end{array} \\
\text { NA }\end{array}$} & \multirow{2}{*}{$\begin{array}{l}\text { Imaging } \\
\\
1.5 \mathrm{~T} \\
\text { MRI }\end{array}$} & \multirow{2}{*}{$\begin{array}{l}\begin{array}{l}\text { Definition } \\
\text { of renal } \\
\text { impairment }\end{array} \\
\text { eGFR as a } \\
\text { continuous } \\
\text { relationship }\end{array}$} & \multirow{2}{*}{$\begin{array}{l}\begin{array}{l}\text { Lacunar } \\
\text { ischaemic } \\
\text { stroke } \\
\text { impaired/total }\end{array} \\
\text { low eGFR correla } \\
\begin{array}{l}\text { WMH volume, } r \\
\mathrm{p}=0.002\end{array}\end{array}$} & \multirow{2}{*}{$\begin{array}{l}\text { Non-lacunar } \\
\text { ischaemic } \\
\text { stroke } \\
\text { impaired/total } \\
\text { ated with } \\
=-0.003,\end{array}$} \\
\hline $\begin{array}{l}\text { Rost } \\
{[12], 2010} \\
\text { USA }\end{array}$ & $\begin{array}{l}\text { Setting: Emergency Department } \\
\text { Included: } 523 \text { consecutive ischaemic stroke patients } \\
\text { Excluded: patients without a lesion on MRI }\end{array}$ & & & & & \\
\hline \multicolumn{7}{|c|}{ Studies of patients with MR imaging features of SVD, but no symptomatic stroke } \\
\hline $\begin{array}{l}\text { Uzu [38], } \\
2010 \\
\text { Japan }\end{array}$ & $\begin{array}{l}\text { Setting: diabetic outpatient clinic } \\
\text { Included: } 608 \text { type } 2 \text { diabetic } \\
\text { Excluded: IHD, cancer, steroid use, heavy proteinuria } \\
300 \mathrm{ml} / \mathrm{l}+\text {, renal impairment }\end{array}$ & $\begin{array}{l}1.5 \mathrm{~T} \\
\text { MRI }\end{array}$ & $\begin{array}{l}1+\mathrm{SBI} \\
\text { definition } \\
\text { not given }\end{array}$ & $\begin{array}{l}\text { micro } \\
\text { albuminuria } \\
(30-299 \mathrm{ml} / \mathrm{l})\end{array}$ & $95 / 177^{2}$ & $188 / 431$ \\
\hline $\begin{array}{l}\text { de Bresser } \\
{[27]} \\
2010 \\
\text { Netherlands }\end{array}$ & $\begin{array}{l}\text { Setting: patients aged } 56-80 \text { with diabetes recruited though } \\
\text { their General Practitioners } \\
\text { Included: } 122 \text { patients with Type } 2 \text { diabetes } \\
\text { Excluded: patients with psychiatric and neurological } \\
\text { disorders, heavy alcohol use and dementia }\end{array}$ & $\begin{array}{l}1.5 \mathrm{~T} \\
\text { MRI }\end{array}$ & $\begin{array}{l}\text { automated } \\
\text { measurement } \\
\text { of WMH } \\
\text { volume }\end{array}$ & $\begin{array}{l}\text { albuminuria } \\
>0.03 \mathrm{~g} / \mathrm{l}\end{array}$ & $\begin{array}{l}\text { baseline albumir } \\
\text { associated with a } \\
\text { significant incre } \\
\text { at } 2 \text { years }\end{array}$ & $\begin{array}{l}\text { nuria was } \\
\text { a non- } \\
\text { ease in WMH }\end{array}$ \\
\hline $\begin{array}{l}\text { Seliger [34], } \\
2005 \\
\text { USA } 40\end{array}$ & $\begin{array}{l}\text { Setting: Cardiovascular Health Study } \\
\text { 5,888 individuals over } 65 \text { selected randomly from medicare } \\
\text { lists } \\
\text { Included: } 2,784 \text { participants selected for MRI } \\
\text { Excluded: previous stroke and TIA }\end{array}$ & not clear & $\begin{array}{l}1+\text { infarct-like } \\
\text { lesion } \geq 3 \mathrm{~mm} \text { in } \\
\text { a patient without } \\
\text { a history of } \\
\text { stroke }\end{array}$ & eGFR $<60$ & $237 / 789$ & $484 / 1,995^{2}$ \\
\hline \multirow[t]{3}{*}{$\begin{array}{l}\text { Wada [39], } \\
2007 ;[40] \\
2008 ;[41] \\
2010 \\
\text { Japan }\end{array}$} & $\begin{array}{l}\text { Setting: population study of all } 61 \text { and } 72 \text { year olds from } \\
\text { two towns } \\
\text { Included: } 608 \text { participants } \\
\text { Excluded: history of stroke, current UTI }\end{array}$ & $\begin{array}{l}0.3 \& \\
0.5 \mathrm{~T} \\
\mathrm{MRI}\end{array}$ & $\begin{array}{l}\text { Fazekas score } \\
\text { of either } 2 \text { or } \\
3 \text { (not specified } \\
\text { whether deep or }\end{array}$ & $\begin{array}{l}\text { presence of } \\
\text { micro } \\
\text { albuminuria: } \\
\text { cut off not clear }\end{array}$ & $95 / 177$ & $188 / 431$ \\
\hline & & & & $\begin{array}{l}\text { eGFR }<60 \text { or } \\
\text { urinary ACR }<30\end{array}$ & $70 / 143$ & $157 / 508$ \\
\hline & & & & Cystatin C & $\begin{array}{l}\text { OR of moderate } \\
\text { WMH, per SD ir } \\
\text { cystine C } 1.48 \text { (1 } \\
\text { (unadjusted) }\end{array}$ & $\begin{array}{l}\text { or severe } \\
\text { ncrease in } \\
1.22-1.78)\end{array}$ \\
\hline $\begin{array}{l}\text { Weiner [42], } \\
2009 \\
\text { USA }\end{array}$ & $\begin{array}{l}\text { Setting: clients of a home care service for low income people } \\
\text { over } 60 \\
\text { Included: } 319 \text { participants } \\
\text { Excluded: participants who were unable to consent, } \\
\text { non-English speakers, had a visual or hearing disability, on } \\
\text { dialysis or unable to provide a urine specimen }\end{array}$ & $\begin{array}{l}1.5 \mathrm{~T} \\
\mathrm{MRI}\end{array}$ & $\begin{array}{l}\text { a score of } 2 / 10 \\
\text { or more on an } \\
\text { unvalidated } \\
\text { qualitative } \\
\text { WMH rating } \\
\text { scale }\end{array}$ & $\begin{array}{l}\text { micro- } \\
\text { albuminuria } \\
(17 \mathrm{mg} / \mathrm{g}+\text { in } \\
\text { men and } \\
25 \mathrm{mg} / \mathrm{g}+\text { in } \\
\text { women })\end{array}$ & $88 / 214$ & $29 / 105$ \\
\hline
\end{tabular}


Table 1. (continued)

\begin{tabular}{|c|c|c|c|c|c|c|}
\hline Study & Patients & Imaging & Definition SVD & $\begin{array}{l}\text { Measure of } \\
\text { renal function }\end{array}$ & $\begin{array}{l}\text { Subjects with } \\
\text { SVD impaired/ } \\
\text { total }\end{array}$ & $\begin{array}{l}\text { Subjects } \\
\text { without SVD } \\
\text { impaired/ } \\
\text { total }\end{array}$ \\
\hline $\begin{array}{l}\text { Otani } \\
{[32]} \\
2010 \\
\text { Japan }\end{array}$ & $\begin{array}{l}\text { Setting: population study of one town } \\
\text { Included: } 1,008 \text { participants aged over } 55 \\
\text { Excluded: previous stroke or TIA }\end{array}$ & $\begin{array}{l}0.5 \mathrm{~T} \\
\mathrm{MRI}\end{array}$ & $\begin{array}{l}\text { at least } 1 \\
\text { hyperintensity } \\
\text { on } \mathrm{T} 2 \text { between } \\
3 \text { and } 15 \mathrm{~mm}\end{array}$ & eGFR $<60$ & $100 / 286$ & $186 / 722$ \\
\hline $\begin{array}{l}\text { Bouchi } \\
{[25]} \\
2010 \\
\text { Japan }\end{array}$ & $\begin{array}{l}\text { Setting: patients with type } 2 \text { diabetes who had an MRI for } \\
\text { any reason at a single hospital } \\
\text { Included: } 786 \text { participants } \\
\text { Excluded: patients with type } 1 \mathrm{DM} \text {, pregnancy, infection, } \\
\text { cancer, or eGFR under } 15\end{array}$ & $\begin{array}{l}1.5 \mathrm{~T} \\
\mathrm{MRI}\end{array}$ & $\begin{array}{l}\text { T2 } \\
\text { hyperintensity } \\
\geq 3 \mathrm{~mm}\end{array}$ & eGFR $<60$ & $182 / 415$ & $75 / 371$ \\
\hline $\begin{array}{l}\text { Chou [26], } \\
2011 \\
\text { Taiwan }\end{array}$ & $\begin{array}{l}\text { Setting: healthy volunteers from Taipai City } \\
\text { Included: } 1,312 \text { participants } \\
\text { Excluded: previous stroke, current fever, eGFR }<30\end{array}$ & $\begin{array}{l}1.5 \mathrm{~T} \\
\mathrm{MRI}\end{array}$ & $\begin{array}{l}\mathrm{T} 2 \\
\text { hyperintensity } \\
\geq 3 \mathrm{~mm}\end{array}$ & eGFR 30-60 & $10 / 62$ & $37 / 1,250$ \\
\hline $\begin{array}{l}\text { Anan }[24] \\
2008 \\
\text { Japan }\end{array}$ & $\begin{array}{l}\text { Setting: outpatient endocrinology clinic } \\
\text { Included: } 90 \text { patients with type } 2 \text { diabetes } \\
\text { Excluded: patients with IHD, macro-albuminuria, high } \\
\text { creatinine, or insulin use }\end{array}$ & $\begin{array}{l}1.5 \mathrm{~T} \\
\mathrm{MRI}\end{array}$ & $\begin{array}{l}\text { the presence of } \\
\text { WMH with } \\
\text { Fazekas score } \\
\geq 2-\text { unclear if } \\
\text { deep or } \\
\text { periventricular }\end{array}$ & $\begin{array}{l}\text { urinary } \\
\text { albumin in the } \\
\text { range of } \\
30-299 \mathrm{mg} / 24 \mathrm{~h}\end{array}$ & $27 / 34$ & $20 / 56$ \\
\hline $\begin{array}{l}\text { Eguchi }[28] \\
2004 \\
\text { Japan }\end{array}$ & $\begin{array}{l}\text { Setting: asymptomatic patients having an annual health } \\
\text { check } \\
\text { Included: } 170 \text { patients aged } 42-89 \text { with } 3 \text { or more vascular } \\
\text { risk factors } \\
\text { Excluded: renal or liver failure, secondary or malignant } \\
\text { hypertension }\end{array}$ & $\begin{array}{l}0.5 \mathrm{~T} \\
\mathrm{MRI}\end{array}$ & $\begin{array}{l}\text { at least } 1 \\
\text { hyperintensity } \\
\text { on } \mathrm{T} 2 \text { between } \\
3 \text { and } 15 \mathrm{~mm}\end{array}$ & $\begin{array}{l}\text { correlation } \\
\text { between serum } \\
\text { creatinine and } \\
\text { no of WMH }\end{array}$ & \multicolumn{2}{|c|}{$\begin{array}{l}\text { serum creatinine correlated } \\
\text { with number of } \mathrm{WMH}, \mathrm{r}=0.2 \text {, } \\
\mathrm{p}<0.006\end{array}$} \\
\hline $\begin{array}{l}\text { Khatri }[31] \\
2007 \\
\text { USA }\end{array}$ & $\begin{array}{l}\text { Setting: randomly selected residents of Manhattan } \\
\text { Included: } 615 \text { participants over } 40 \text { who had a telephone } \\
\text { and could consent } \\
\text { Excluded: those with a history of stroke or eGFR }<15\end{array}$ & $\begin{array}{l}1.5 \mathrm{~T} \\
\mathrm{MRI}\end{array}$ & $\begin{array}{l}\text { automated } \\
\text { measurement } \\
\text { of WMH } \\
\text { volume }\end{array}$ & $\begin{array}{l}\text { correlation } \\
\text { between } \\
\text { creatinine } \\
\text { clearance and } \\
\text { WMH volume }\end{array}$ & \multicolumn{2}{|c|}{$\begin{array}{l}\text { creatinine clearance of } 15-60 \\
\text { ml/linked to log WMH volume } \\
(0.322 ; 95 \% \mathrm{CI}, 0.095-0.550)\end{array}$} \\
\hline \multirow[t]{2}{*}{$\begin{array}{l}\text { Takahashi } \\
{[35]} \\
2012 \\
\text { Japan }\end{array}$} & \multirow[t]{2}{*}{$\begin{array}{l}\text { Setting: asymptomatic patients presenting for a 'brain } \\
\text { check'. } \\
\text { Included: } 2,043 \text { healthy volunteers. } \\
\text { Excluded: participants with a history of stroke, neurological, } \\
\text { or heart diseases }\end{array}$} & \multirow[t]{2}{*}{$\begin{array}{l}1.5 \mathrm{~T} \\
\mathrm{MRI}\end{array}$} & \multirow[t]{2}{*}{$\begin{array}{l}\text { score of } \geq 2 \text { on } \\
\text { Fazekas score - } \\
\text { deep and } \\
\text { periventricular } \\
\text { lesions analysed } \\
\text { separately }\end{array}$} & \multirow[t]{2}{*}{ eGFR $<60$} & $\begin{array}{l}\text { deep WMH } \\
89 / 465\end{array}$ & $\begin{array}{l}\text { deep WMH } \\
156 / 1,571\end{array}$ \\
\hline & & & & & $\begin{array}{l}\text { periventricular } \\
\text { WMH: } \\
51 / 221\end{array}$ & $\begin{array}{l}\text { periventricular } \\
\text { WMH: } \\
\text { 194/1,822 }\end{array}$ \\
\hline \multirow[t]{2}{*}{$\begin{array}{l}\text { Takami [36], } \\
2012 \\
\text { Japan }\end{array}$} & \multirow[t]{2}{*}{$\begin{array}{l}\text { Setting: outpatient hyperten } \\
\text { Included: } 185 \text { participants } \\
\text { Excluded: patients with AF }\end{array}$} & \multirow[t]{2}{*}{$\begin{array}{l}1.5 \mathrm{~T} \\
\mathrm{MRI}\end{array}$} & \multirow[t]{2}{*}{$\begin{array}{l}\text { deep WMH: } \\
\text { cases if Fazekas } \\
\text { score } \geq 2 \text {. } \\
\text { Periventricular } \\
\text { WMH cases if } \\
\text { Fazekas score } \geq 1\end{array}$} & eGFR $<60$ & $\begin{array}{l}\text { deep WMH } \\
31 / 75\end{array}$ & $\begin{array}{l}\text { deep WMH } \\
16 / 110^{2}\end{array}$ \\
\hline & & & & & $\begin{array}{l}\text { periventricular } \\
\text { WMH } \\
36 / 102\end{array}$ & $\begin{array}{l}\text { periventricular } \\
\text { WMH } \\
10 / 83^{2}\end{array}$ \\
\hline $\begin{array}{l}\text { Turner [37], } \\
2011 \\
\text { USA }\end{array}$ & $\begin{array}{l}\text { Setting: members of sibling pairs where one was } \\
\text { hypertensive } \\
\text { Included: } 1,585 \text { participants } \\
\text { Excluded: secondary hwypertension, known CKD and } \\
\text { previous stroke }\end{array}$ & $\begin{array}{l}1.5 \mathrm{~T} \\
\mathrm{MRI}\end{array}$ & $\begin{array}{l}\text { automated } \\
\text { measurement } \\
\text { of WMH } \\
\text { volume on } \\
\text { FLAIR }\end{array}$ & $\begin{array}{l}\text { correlation } \\
\text { between serum } \\
\text { creatinine and } \\
\text { WMH volume }\end{array}$ & \multicolumn{2}{|c|}{$\begin{array}{l}\text { correlation between serum } \\
\text { creatinine and WMH volume. } \\
\text { Age adjusted correlation } \\
\text { coefficient }=0.54\end{array}$} \\
\hline
\end{tabular}


Table 1. (continued)

\begin{tabular}{|c|c|c|c|c|c|c|}
\hline Study & Patients & Imaging & Definition SVD & $\begin{array}{l}\text { Measure of } \\
\text { renal function }\end{array}$ & $\begin{array}{l}\text { Subjects with } \\
\text { SVD impaired/ } \\
\text { total }\end{array}$ & $\begin{array}{l}\text { Subjects } \\
\text { without SVD } \\
\text { impaired/ } \\
\text { total }\end{array}$ \\
\hline $\begin{array}{l}\text { Ravera } \\
\text { [33], } 2002 \\
\text { Italy }\end{array}$ & $\begin{array}{l}\text { Setting: patients from one centre who were enrolled in a } \\
\text { large study on complications of microalbuminuria in } \\
\text { untreated patients with mild-moderate hypertension } \\
\text { Included: } 22 \text { patients with microalbuminuria, } 22 \text { controls } \\
\text { without } \\
\text { Excluded: patients with cancer, liver disease, IHD, diabetes, } \\
\text { obesity, and Dementia }\end{array}$ & $\begin{array}{l}1.5 \mathrm{~T} \\
\text { MRI }\end{array}$ & $\begin{array}{l}\text { a count of } \\
\text { number of } \\
\text { lacuanes: } 3-15 \\
\text { mm lesion dark } \\
\text { on T1, light on } \\
\text { T2 }\end{array}$ & \multicolumn{3}{|c|}{$\begin{array}{l}\text { no of lacunes in } 11 \text { patients with } \\
\text { microalbuminuria against the number of lacunas } \\
\text { in } 11 \text { patients without microalbuminuria } 82 \text { of } \\
\text { patients with microalbuminuria had incident } \\
\text { lacunes vs. } 27 \% \text { of patients without }\end{array}$} \\
\hline
\end{tabular}

\footnotetext{
${ }^{1}$ All studies excluded patients unable to have MRI; ${ }^{2}$ Calculated from mean and SD assuming a normal distribution. UTI = Urinary tract infection; TOAST = trial of org 10,172 in acute stroke treatment; $\mathrm{CKD}=$ chronic kidney disease; $\mathrm{CT}=$ computerised topography; $\mathrm{MRI}=$ magnetic resonance imaging; $\mathrm{OCSP}=$ oxfordshire community stroke project; $\mathrm{eGFR}=$ estimated glomular filtration rate; TPA $=$ tissue plasminogen antigen; $\mathrm{WMH}=$ white matter hyperintensities; $\mathrm{SD}=$ standard deviation; $\mathrm{IHD}=$ ischaemic heart disease; TIA = transient ischaemic attack; CSF = cerebrospinal fluid; $\mathrm{OR}=$ odds ratio; $\mathrm{ACR}=$ albumin creatinine ratio.
}

23] measured eGFR. No studies reported whether there were any differences in vascular risk factors between patients with lacunar and non-lacunar stroke.

\section{Characteristics of 17 Studies of Non-Stroke Patients}

Seventeen studies [24-42, 46, 47] of 13,164 participants (mean age 65) measured renal function and MRI features of SVD in participants without a symptomatic stroke (tables 1 and 2). Eight studies [26, 30-35, 39] were of healthy volunteers; four were of diabetic patients $(10 \%$ of subjects); two $[28,29]$ were of patients with any of a variety of vascular risk factors, one was of hypertensive patients alone [36], and another included both hypertensive patients and their siblings (12\% of subjects) [34, 37].

Ten studies [24-26, 28, 29, 32, 34, 36, 38, 39] reported the blood pressure of participants with and without SVD (either as the percent of subjects previously diagnosed with hypertension or as the mean systolic blood pressure on examination), and two studies $[24,36]$ reported little difference between the participants with and without SVD (a difference of less than 2\% in the proportion of participants or a difference of less than $5 \mathrm{~mm} \mathrm{Hg}$ in systolic blood pressure between groups).

Ten studies [26, 28, 29, 31, 32, 34, 37-39] excluded participants with previous stroke, nine [24-26, 28, 31, 33, 38, 39,42 ] excluded those with the most severe renal impairment, and all measured the MRI features of SVD.

Five studies $[24,28,35,36,38,40]$ used a method such as the Fazekas score [48] to grade WMH, six [25, 26, 29, $32,34,38$ ] counted the number of silent brain infarcts, and five $[27,30,31,37,42]$ used an automated measure to quantify the volume of WMH. Nine studies [24, 26-29,
$33,34,36,41,42]$ ( $46 \%$ of participants) were read by an observer blinded to the clinical details. Eight studies [26, $27,30,31,34-37$ ] (70\% of non-stroke participants) measured eGFR, six $[24,25,33,38,39,42]$ measured proteinuria, three $[28,32,40]$ measured both, and one study [29] measured serum creatinine. Thirteen studies [24-32, 34$36,39,42$ ] carried out a multivariate analysis accounting for (at least) age and hypertension. Twelve papers treated SVD as a binary variable (i.e., present or absent) and performed binary logistic regression, while seven treated it as a continuous variable and used linear regression.

\section{Meta-Analysis of the Risk of Renal Impairment in \\ Lacunar Stroke Versus Other Stroke Subtypes}

First, we performed a meta-analysis of the studies reporting the numbers of lacunar and non-lacunar stroke patients with renal impairment (defined as proteinuria or an eGFR below $60 \mathrm{ml} / \mathrm{min}$ ): 12 studies of 5,338 patients $[9,10,13-18,20-23]$. We excluded a study of patients who suffered deterioration in renal function after stroke [19] and two studies of stroke patients that measured WMH volume, not stroke subtype $[11,12]$.

Overall there was no specific association between renal function and stroke subtype - lacunar versus non-lacunar: $394 / 1,119$ (35\%) of patients with lacunar stroke had renal impairment, compared with 1,443/4,217 (34\%) of patients with non-lacunar stroke (fig. 2) OR $0.88,95 \%$ confidence interval (CI) $0.61-1.28$. There was a high degree of heterogeneity (inconsistency) with an $\mathrm{I}^{2}$ of $76 \%$. When comparing patients with lacunar and non-lacunar stroke there was no statistically significant difference in the odds of proteinuria, OR 0.79 (95\% CI $0.38-1.67$ ), or eGFR $<60$, 
Table 2. Summary of characteristics of studies

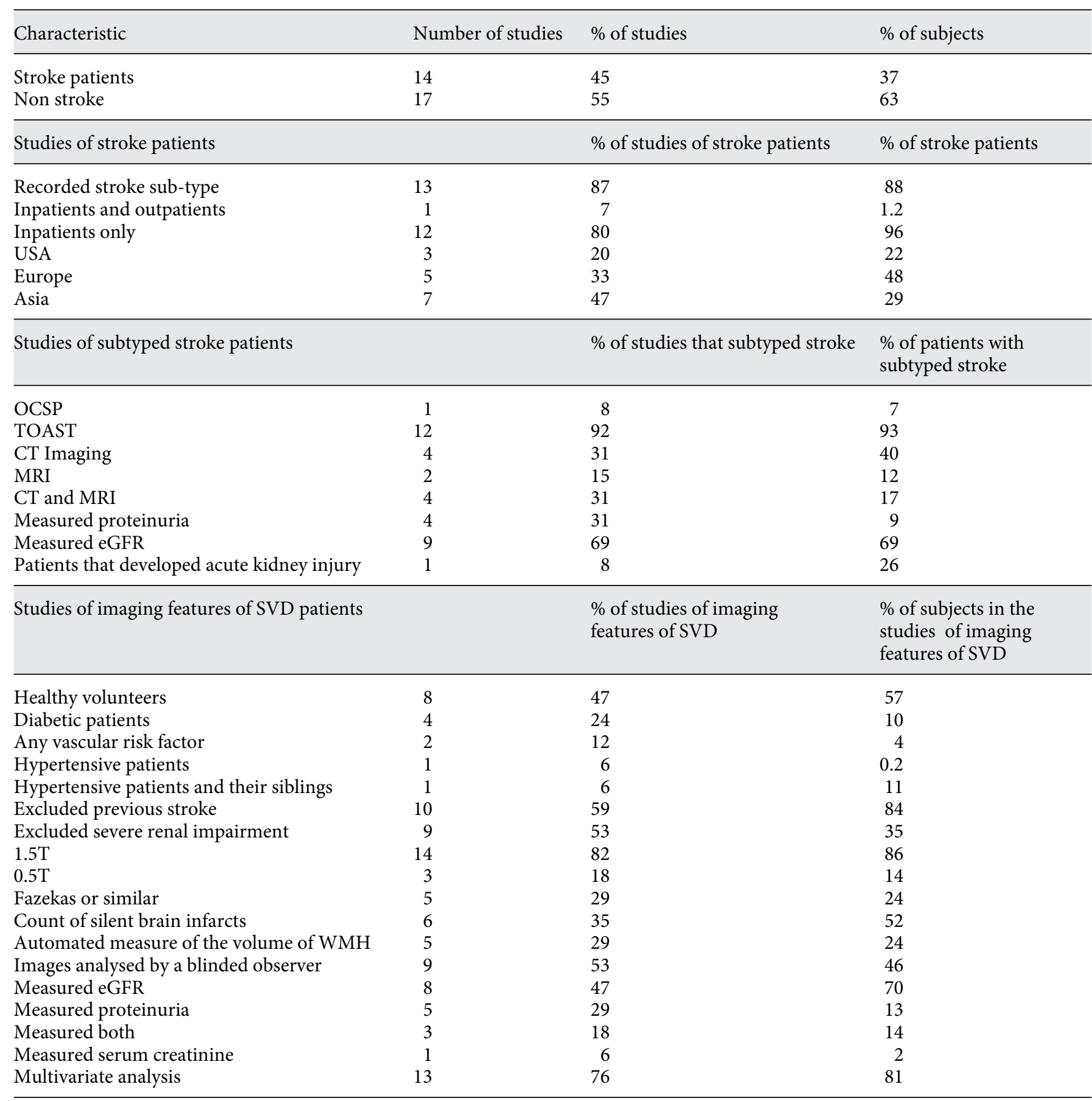

OR 1.02 (95\% CI: 0.66-1.56). No studies of stroke patients performed a multivariable analysis accounting for risk factors. Funnel plots (online suppl. fig. A, B; see www. karger.com/doi/10.1159/000369777) did not indicate publication bias.
Sub-group analysis (fig. 3) suggested an association between lacunar stroke and impaired renal function in younger patients: a study that only included participants aged 15-49 [20] found a fourfold risk of renal impairment when compared to other subtypes, OR 4.64 (95\% CI: 


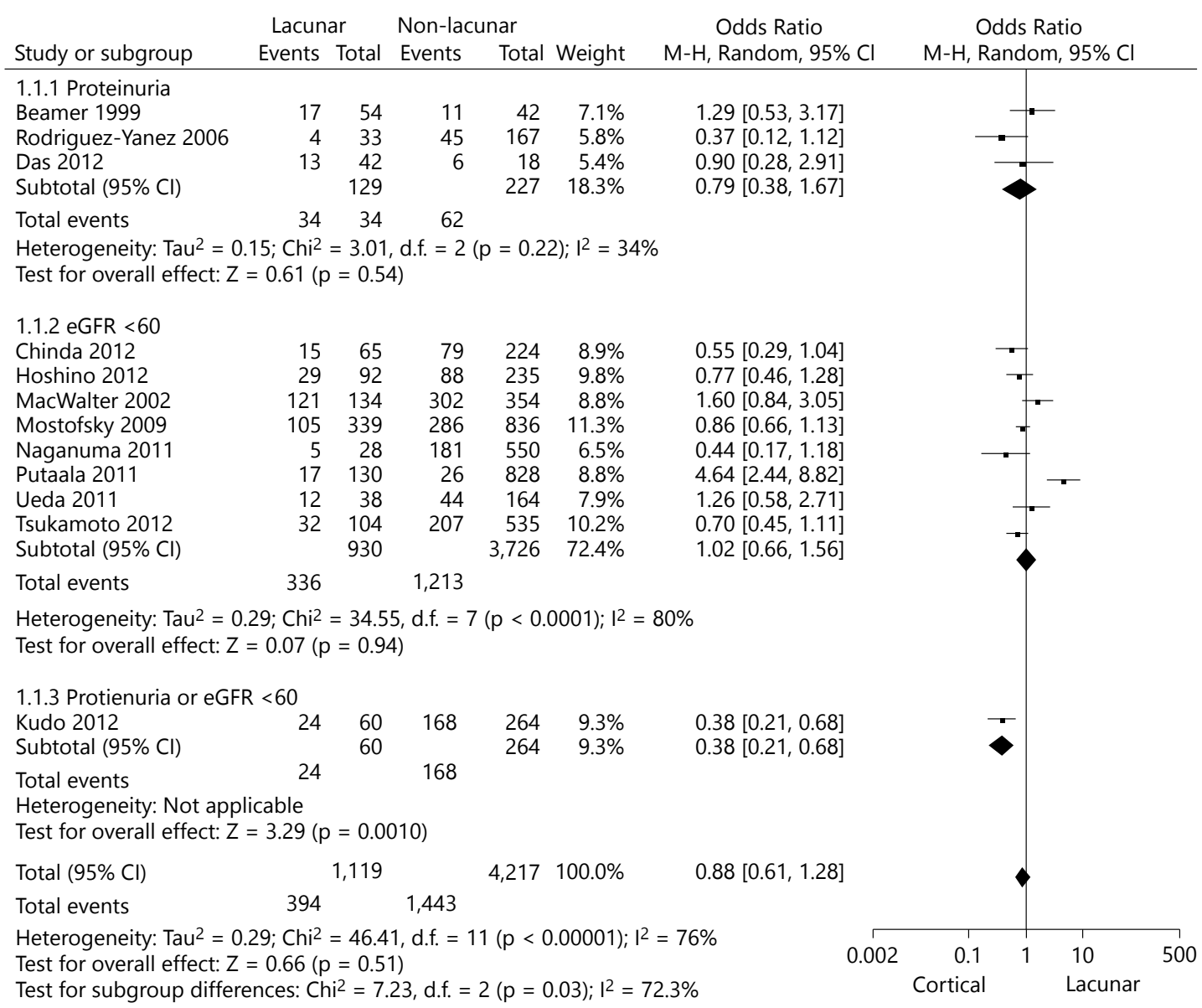

Fig. 2. A forest plot demonstrating the results of the meta-analysis of studies of renal function in patients with lacunar and cortical stroke.

Fig. 3. The Odds Ratio of renal impairment in patients with lacunar stroke compared to other stroke sub-type for different subgroups of studies.

Renal Function and Small Vessel Disease

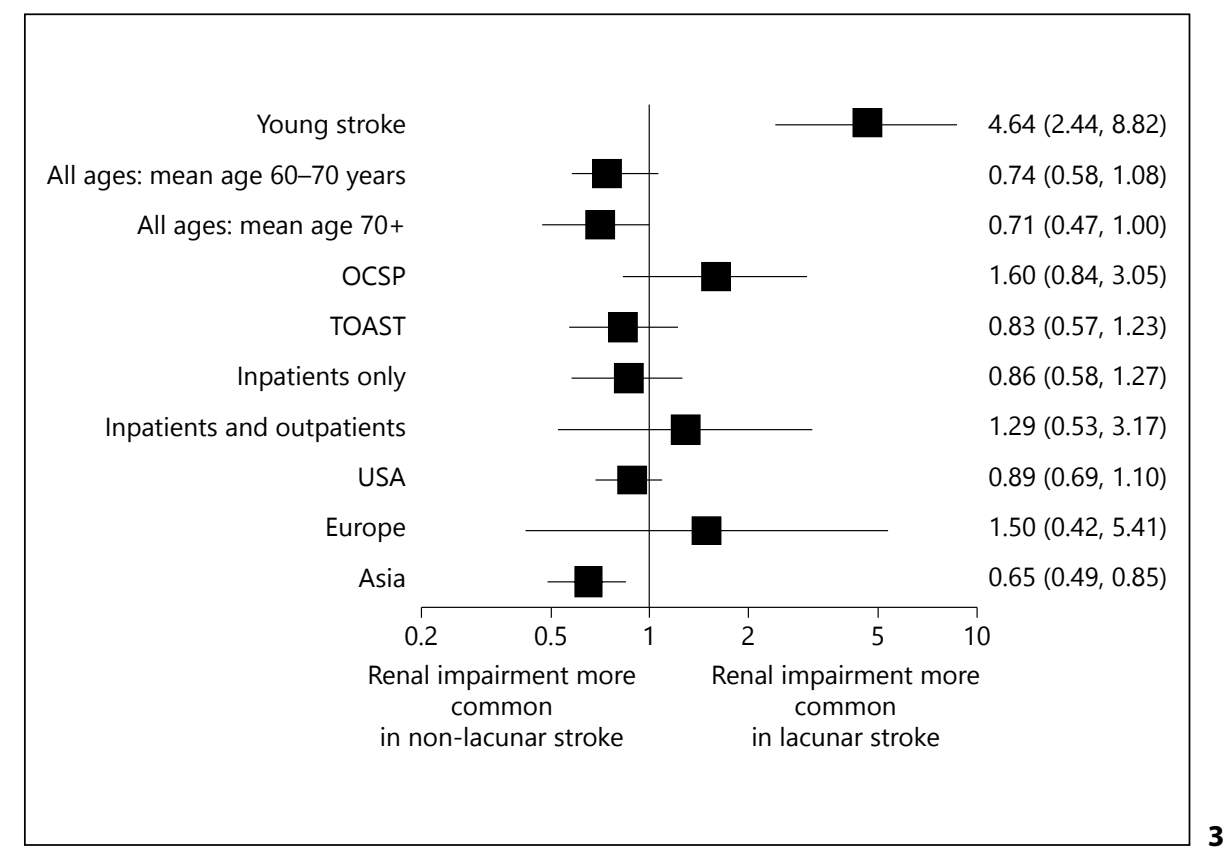

Cerebrovasc Dis 2015;39:39-52 DOI: $10.1159 / 000369777$ 


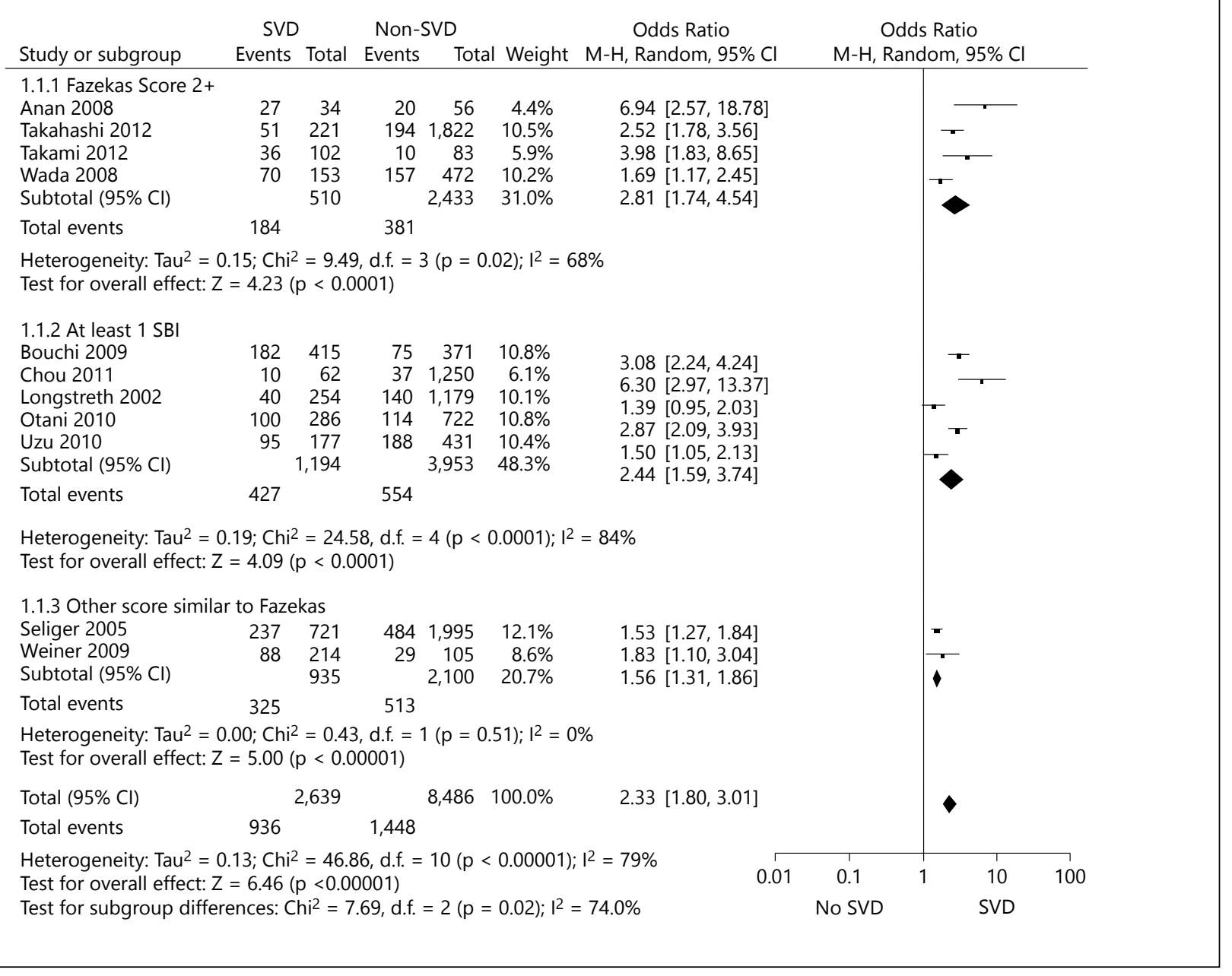

Fig. 4. Meta-analysis of the risk of renal impairment in non-stroke patients with SVD compared to those without SVD.

2.44-8.82); whereas in studies of patients with a mean age of 70 or greater there was no significant difference between subtypes. In Asian studies (Japan [9, 15, 17, 21-23], and Bangladesh [10]) patients with lacunar stroke had a reduced risk of renal impairment OR 0.65 (95\% CI 0.490.85). Neither the method of stroke sub-typing (OCSP or TOAST), nor a study that recruited only inpatients, affected the lack of association between lacunar stroke subtype and renal impairment.

\section{Meta-Analysis of the Risk of Renal Impairment in \\ Non-Stroke Participants}

We included 12 studies [24-26, 32, 34-36, 38-40, 42, 46] of 11,269 participants in this analysis. We excluded studies that only measured Cystatin C [41] or serum creatinine [29]; those that used microalbuminuria as the dependent variable [33]; or those that did not dichotomise renal impairment or SVD [30, 31, 37, 47]. For the two studies [35, 36] that did not report a total WMH score but instead reported the findings for deep and periventricular WMH separately, we included the data for periventricular lesions in the meta-analysis, as these are more prevalent. Two studies reported the results of eGFR and proteinuria in separate papers [32, 39, 40, 49]; therefore, we ensured that each participant only contributed once to each calculation.

\section{Unadjusted Odds of Renal Impairment in Participants with and without Imaging Features of Small Vessel \\ Disease}

The OR of renal impairment (either eGFR or proteinuria) in participants with SVD compared to those without was 2.33 (95\% CI 1.80-3.01), with an $\mathrm{I}^{2}$ of $78.2 \%$ (fig. 4). 


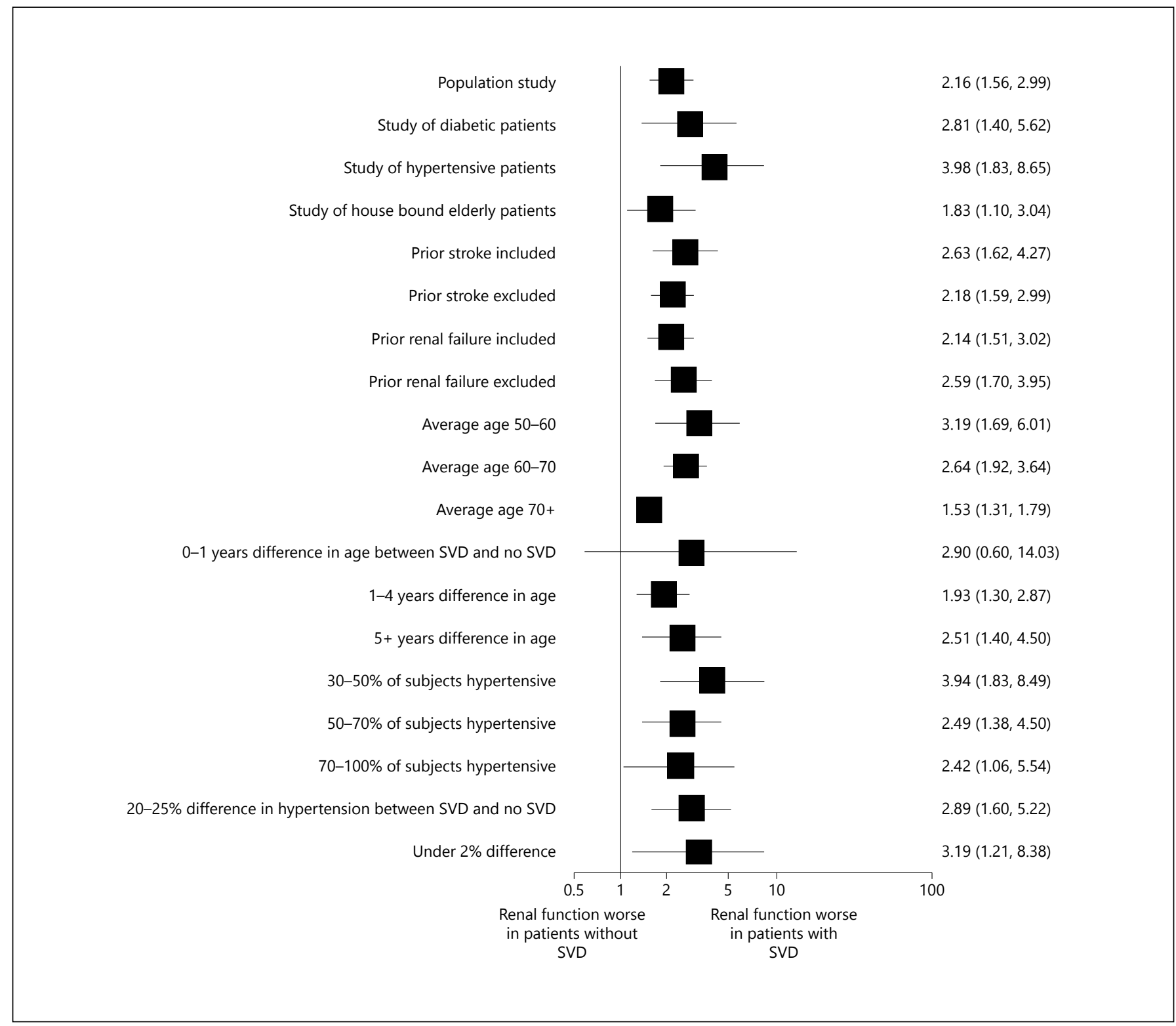

Fig. 5. The Odds Ratio of renal impairment in non-stroke patients with small vessel disease compared to those without for different subgroups of studies.

Studies that recorded 'silent brain infarcts' had a higher degree of heterogeneity ( $\left.\mathrm{I}^{2} 84 \%\right)$, which may represent the range of different lesions described as 'silent brain infarcts'. Further meta-analysis of the unadjusted data revealed that participants with SVD were twice as likely to have proteinuria compared with participants without SVD: OR 2.00 (95\% CI 1.44-2.78) with a moderate degree of heterogeneity ( $\left.\mathrm{I}^{2} 54.1 \%\right)$; and almost three times as likely to have an eGFR $<60$, OR 2.82 (95\% CI 1.94-4.10), but with a high degree of heterogeneity: $\mathrm{I}^{2} 84.4 \%$.

Renal Function and Small Vessel Disease
We investigated whether the relationship between SVD and renal function varied in studies of particular groups of participants (fig. 5). For studies of younger patients (average age 50-60) there was a stronger relationship between impaired renal function and SVD (OR 3.19, 95\% CI 1.69-6.01) in comparison with studies of patients over 70 (OR 1.53, 95\% CI 1.53-1.79). Other study factors had little consistent effect on the relationship between renal function and SVD. A funnel plot (online suppl. fig. B) revealed little evidence of publication bias. 
Risk Factor Adjusted Odds of Renal Impairment in

Participants with and without Imaging Features of

Small Vessel Disease

Nineteen studies [24-32, 34-37, 39, 40, 42, 46, 47, 49]

calculated odds ratios adjusted for age, hypertension and a variety of other risk factors; but we could not carry out a meta-analysis of the adjusted ORs as all studies adjusted for slightly different parameters.

After adjusting for (at least) age and hypertension, nine studies [24-27, 31, 35, 42, 46, 47] reported a significant association between renal function and SVD, but with a smaller OR than the unadjusted statistic. However, three studies $[28,34,37]$ reported no significant link between renal function and SVD after adjustment for age and hypertension; one [49] found a significant link between proteinuria and SVD, but not between eGFR and SVD; one [30] found a significant link between renal impairment for participants with periventricular, but not deep $\mathrm{WMH}$, whereas another [36] found that there was a significant link between renal impairment and deep (but not periventricular) $\mathrm{WMH}$.

\section{Discussion}

Participants with cerebral SVD features on imaging were found to be at increased risk of renal impairment compared to participants without SVD, but patients with a symptomatic lacunar stroke were at no more risk of renal impairment than patients with a non-lacunar stroke.

An association between lacunar sub-type and renal impairment could have been missed by the studies of stroke patients because the sub-typing (largely based on clinical and CT diagnosis) was not sufficiently precise to distinguish lacunar from non-lacunar stroke [50]. No studies used a gold-standard means of stroke sub-typing, namely risk-factor free clinical sub-typing aided by DWI-MRI in the acute phase. As some studies [51] have suggested that lacunar stroke affects patients at a younger age than non-lacunar stroke, the lack of adjustment for age in the analysis of patients with symptomatic stroke may have masked an association between lacunar stroke subtype and impaired renal function. In a study of younger patients [20] there was a stronger association between impaired renal function and lacunar stroke than with other stroke subtypes, which is interesting as an association between renal function and lacunar stroke may not be present across all age groups as it could be diluted by older patients having more heterogeneous risk factors.
We did not investigate whether the different non-lacunar sub-types were associated with renal impairment as we were investigating the association between small vessel disease and renal impairment.

There was a high level of heterogeneity throughout the literature with different methods of measuring SVD, stroke sub-type, proteinuria, and eGFR. No studies reported differences in risk factors between patients with and without lacunar stroke, which limited our investigation of covariates such as hypertension. Almost all studies measured renal impairment in the acute phase, leading to potential confounding by dehydration, which is common after stroke [52].

This work has been hampered by the lack of a standardised definition of SVD, with various studies using definitions such as silent brain infarcts, and 'lacunes' to represent similar imaging findings. This problem was compounded by various definitions of proteinuria/albuminuria. Future studies should use the recently published standardised imaging definitions of SVD [7].

The strengths of this review include a comprehensive literature search incorporating studies from America, Europe and Asia with no language exclusions. In addition to the relationship between reduced eGFR and silent lesions investigated by Vogels et al. [2] we have included patients with proteinuria, symptomatic stroke, and a meta-analysis. Weaknesses comprise the inclusion of only dichotomised studies in the meta-analysis: some more recent studies investigated the continuous relationship between WMH volume and renal impairment. We were not able to fully investigate the effects of powerful confounding factors (e.g. age and hypertension), because it was not possible to carry out a meta-analysis of the adjusted ORs from multivariate analysis as they had all corrected for different confounders.

The apparently strong link between 'silent' SVD and renal impairment in studies of stroke-free patients was not seen in studies of symptomatic stroke. This calls into question the hypothesis that cerebral and renal SVD are directly associated as part of the same multi-system disease rather than representing end organ damage from shared risk factors particularly of hypertension. However, it is difficult to draw firm conclusions due to a high degree of heterogeneity and imprecise stroke sub-typing.

Studies of non-stroke participants should use agematched controls and carry out multivariate analysis of confounding factors. As over 10,000 participants have already undergone MRI and the measurement of renal function, it should be possible to achieve this by re-anal- 
ysis of the existing data using a well-resourced individual patient data meta-analysis. Future studies of stroke patients should perform careful sub-typing using risk factor-free clinical classification (i.e. OCSP [44] aided by early DWI-MRI), measure proteinuria and eGFR outside the acute phase, and compare with age-matched non-lacunar stroke controls accounting for variations in risk factors.

\section{Acknowledgements}

We thank Dr. K. Konta [18] Dr. Nakagawa [11] and Dr. K. Ueda [37] for providing extra information to enable their papers to be used in the meta-analysis, and Ms. C. Chapman for proof reading the final manuscript.

The authors made the following contributions to the paper: S.D.J. Makin: Designed the protocol, carried out search, extracted data, performed the statistical analysis and drafted the manuscript.

F.A.B. Cook: Carried out search and extracted the data.
M.S. Dennis: Discussed the design of the project, discussed and reviewed the manuscript.

J.M. Wardlaw: Conceived the project, drafted the protocol, amended the design, reviewed potentially suitable papers, edited drafts, reviewed the final manuscript and data analysis.

Search terms: Lacunar Stroke, Infarction Stroke, Small Vessel Disease, MRI, Kidney.

\section{Funding}

S.D.J.M. is supported by a Wellcome Trust Project Grant (WT088134/Z/09/A). J.M.W. is supported by the Scottish Funding Council through the Scottish Imaging Network, A Platform for Scientific Excellence (SINAPSE) Initiative (http://www.sinapse. ac.uk). The study was independent of the funders.

\section{Disclosure Statement}

The authors have no conflict of interest.

\section{References}

$\checkmark 1$ Lee M, Saver JL, Chang KH, Liao HW, Chang SC, Ovbiagele B: Low glomerular filtration rate and risk of stroke: meta-analysis. BMJ 2010;341:c4249.

2 Vogels SC, Emmelot-Vonk MH, Verhaar HJ, Koek HL: The association of chronic kidney disease with brain lesions on MRI or CT: a systematic review. Maturitas 2012;71:331336.

3 Thompson CS, Hakim AM: Living beyond our physiological means: small vessel disease of the brain is an expression of a systemic failure in arteriolar function: a unifying hypothesis. Stroke 2009;40:e322-e330.

4 Wiseman S, Marlborough F, Doubal F, Webb DJ, Wardlaw J: Blood markers of coagulation, fibrinolysis, endothelial dysfunction and inflammation in lacunar stroke versus non-lacunar stroke and non-stroke: systematic review and meta-analysis. Cerebrovasc Dis 2014;37:64-75.

5 Lavallee PC, Labreuche J, Faille D, Huisse MG, Nicaise-Roland P, Dehoux M, GongoraRivera F, Jaramillo A, Brenner D, Deplanque D, Klein IF, Touboul PJ, Vicaut E, Ajzenberg $\mathrm{N}$ : Circulating markers of endothelial dysfunction and platelet activation in patients with severe symptomatic cerebral small vessel disease. Cerebrovasc Dis 2013;36:131-138.

-6 Moher D, Liberati A, Tetzlaff J, Altman DG: Preferred reporting items for systematic reviews and meta-analyses: the PRISMA Statement. Open Med 2009;3:e123-e130.

7 Wardlaw JM, Smith EE, Biessels GJ, Cordonnier C, Fazekas F, Frayne R, Lindley RI, O'Brien JT, Barkhof F, Benavente OR, Black SE, Brayne C, Breteler M, Chabriat H, DeCar- li C, de Leeuw FE, Doubal F, Duering M, Fox NC, Greenberg S, Hachinski V, Kilimann I, Mok V, Oostenbrugge R, Pantoni L, Speck O, Stephan BC, Teipel S, Viswanathan A, Werring $D$, Chen $C$, Smith $C$, van Buchem $M$, Norrving B, Gorelick PB, Dichgans M: Neuroimaging standards for research into small vessel disease and its contribution to ageing and neurodegeneration. Lancet Neurol 2013; 12:822-838.

8 Potter GM, Marlborough FJ, Wardlaw JM: Wide variation in definition, detection, and description of lacunar lesions on imaging. Stroke 2011;42:359-366.

$\checkmark 9$ Ueda K, Watanabe Y, Katsumata T, Kaneko $\mathrm{T}$, Otori T, Utsumi K, Iino Y, Katayama Y: Carotid intima-media thickness and cerebral white matter lesions are more advanced in acute ischemic stroke patients with renal dysfunction. Clin Nephrol 2011;76:290-295.

10 Das P, Bhuiyan MM, Mia BA, Ullah MA, Alam SM, Sheikh AK, Islam MR, Islam MS, Rahman HZ: Urinary microalbumin as a risk factor for ischaemic stroke. Mymensingh Med J 2012;21:709-714.

11 Oksala NK, Salonen T, Strandberg T, Oksala A, Pohjasvaara T, Kaste M, Karhunen PJ, Erkinjuntti T: Cerebral small vessel disease and kidney function predict long-term survival in patients with acute stroke. Stroke 2010;41:1914-1920.

-12 Rost NS, Rahman R, Sonni S, Kanakis A, Butler C, Massasa E, Cloonan L, Gilson A, Delgado $\mathrm{P}$, Chang $\mathrm{Y}$, Biffi A, Jimenez-Conde J, Besanger A, Silva G, Smith EE, Rosand J, Furie KL: Determinants of white matter hyperintensity volume in patients with acute ischemic stroke. J Stroke Cerebrovasc Dis 2010;19:230235.

13 Rodriguez-Yanez M, Castellanos M, Blanco M, Millan M, Nombela F, Sobrino T, Lizasoain I, Leira R, Serena J, Davalos A, Castillo $\mathrm{J}$ : Micro- and macroalbuminuria predict hemorrhagic transformation in acute ischemic stroke. Neurology 2006;67:1172-1177.

14 Beamer NB, Coull BM, Clark WM, Wynn M: Microalbuminuria in ischemic stroke. Arch Neurol 1999;56:699-702.

15 Chinda J, Nakagawa N, Kabara M, Matsuki M, Endo H, Saito T, Sawada J, Katayama T, Sato N, Hasebe N: Impact of decreased estimated glomerular filtration rate on Japanese acute stroke and its subtype. Intern Med 2012; 51:1661-1666

16 MacWalter RS, Wong SY, Wong KY, Stewart G, Fraser CG, Fraser HW, Ersoy Y, Ogston SA, Chen R: Does renal dysfunction predict mortality after acute stroke? A 7-year followup study. Stroke 2002;33:1630-1635.

17 Hoshino H, Itoh Y, Yamada S, Miyaki K, Suzuki N: Clinical features and neurologic severity in stroke patients with mild to moderate renal dysfunction. J Stroke Cerebrovasc Dis 2012;21:343-349.

18 Mostofsky E, Wellenius GA, Noheria A, Levi$\tan$ EB, Burger MR, Schlaug G, Mittleman MA: Renal function predicts survival in patients with acute ischemic stroke. Cerebrovasc Dis 2009;28:88-94.

19 Tsagalis G, Akrivos T, Alevizaki M, Manios E, Theodorakis M, Laggouranis A, Vemmos $\mathrm{KN}$ : Long-term prognosis of acute kidney injury after first acute stroke. Clin J Am Soc Nephrol 2009;4:616-622. 
20 Putaala J, Haapaniemi E, Gordin D, Liebkind R, Groop PH, Kaste M, Tatlisumak T: Factors associated with impaired kidney function and its impact on long-term outcome in young ischemic stroke. Stroke 2011;42:2459-2464.

21 Naganuma M, Koga M, Shiokawa Y, Nakagawara J, Furui E, Kimura K, Yamagami H, Okada Y, Hasegawa Y, Kario K, Okuda S, Nishiyama K, Minematsu K, Toyoda K: Reduced estimated glomerular filtration rate is associated with stroke outcome after intravenous rt-PA: the Stroke Acute Management with Urgent Risk-Factor Assessment and Improvement (SAMURAI) rt-PA registry. Cerebrovasc Dis 2011;31:123-129.

$\checkmark 22$ Kudo K, Konta T, Degawa N, Saito S, Kondo R, Kayama T, Kubota I: Relationship between kidney damage and stroke types in Japanese patients. Clin Exp Nephrol 2012;16:564-569.

-23 Tsukamoto Y, Takahashi W, Takizawa S, Kawada S, Takagi S: Chronic kidney disease in patients with ischemic stroke. J Stroke Cerebrovasc Dis 2012;21:547-550.

-24 Anan F, Masaki T, Iwao T, Eto T, Shimomura T, Umeno Y, Eshima N, Saikawa T, Yoshimatsu $\mathrm{H}$ : The role of microalbuminuria and insulin resistance as significant risk factors for white matter lesions in Japanese type 2 diabetic patients. Curr Med Res Opin 2008;24: 1561-1567.

-25 Bouchi R, Babazono T, Yoshida N, Nyumura I, Toya K, Hayashi T, Hanai K, Tanaka N, Ishii A, Iwamoto Y: Relationship between chronic kidney disease and silent cerebral infarction in patients with Type 2 diabetes. Diabet Med 2010;27:538-543.

26 Chou CC, Lien LM, Chen WH, Wu MS, Lin SM, Chiu HC, Chiou HY, Bai CH: Adults with late stage 3 chronic kidney disease are at high risk for prevalent silent brain infarction: a population-based study. Stroke 2011;42: 2120-2125.

-27 de Bresser J, Reijmer YD, van den Berg E, Breedijk MA, Kappelle LJ, Viergever MA, Biessels GJ: Microvascular determinants of cognitive decline and brain volume change in elderly patients with type 2 diabetes. Dement Geriatr Cogn Disord 2010;30:381-386.

28 Eguchi K, Kario K, Hoshide S, Hoshide Y, Ishikawa J, Morinari M, Hashimoto T, Shimada K: Smoking is associated with silent cerebrovascular disease in a high-risk Japanese community-dwelling population. Hypertens Res 2004;27:747-754.

29 Giele JL, Witkamp TD, Mali WP, van der Graaf Y: Silent brain infarcts in patients with manifest vascular disease. Stroke 2004;35: 742-746.

30 Ikram MA, Vernooij MW, Hofman A, Niessen WJ, van der Lugt A, Breteler MM: Kidney function is related to cerebral small vessel disease. Stroke 2008;39:55-61.
Khatri M, Wright CB, Nickolas TL, Yoshita M, Paik MC, Kranwinkel G, Sacco RL, DeCarli C: Chronic kidney disease is associated with white matter hyperintensity volume: the Northern Manhattan Study (NOMAS). Stroke 2007;38:3121-3126.

32 Otani H, Kikuya M, Hara A, Terata S, Ohkubo T, Kondo T, Hirose T, Obara T, Metoki $\mathrm{H}$, Inoue $\mathrm{R}$, Asayama $\mathrm{K}$, Kanno A, Terawaki $\mathrm{H}$, Nakayama M, Totsune K, Hoshi H, Satoh H, Izumi S, Imai Y: Association of kidney dysfunction with silent lacunar infarcts and white matter hyperintensity in the general population: the Ohasama study. Cerebrovasc Dis 2010;30:43-50.

33 Ravera M, Ratto E, Vettoretti S, Viazzi F, Leoncini G, Parodi D, Tomolillo C, Del Sette M, Maviglio N, Deferrari G, Pontremoli R: Microalbuminuria and subclinical cerebrovascular damage in essential hypertension. J Nephrol 2002;15:519-524.

34 Seliger SL, Longstreth WT Jr, Katz R, Manolio T, Fried LF, Shlipak M, Stehman-Breen CO, Newman A, Sarnak M, Gillen DL, Bleyer A, Siscovick DS: Cystatin $\mathrm{C}$ and subclinical brain infarction. J Am Soc Nephrol 2005; 16:3721-3727.

35 Takahashi W, Tsukamoto Y, Takizawa S, Kawada S, Takagi S: Relationship between chronic kidney disease and white matter hyperintensities on magnetic resonance imaging. J Stroke Cerebrovasc Dis 2012;21:18-23.

-36 Takami T, Yamano S, Okada S, Sakuma M, Morimoto T, Hashimoto H, Somekawa S, Saito Y: Major risk factors for the appearance of white-matter lesions on MRI in hypertensive patients with controlled blood pressure. Vasc Health Risk Manag 2012;8:169-176.

-37 Turner ST, Rule AD, Schwartz GL, Kullo IJ, Mosley TH, Jack CR, Kardia SL, Boerwinkle E, Bailey KR: Risk factor profile for chronic kidney disease is similar to risk factor profile for small artery disease. J Hypertens 2011;29: 1796-1801.

38 Uzu T, Kida Y, Shirahashi N, Harada T, Yamauchi A, Nomura M, Isshiki K, Araki S, Sugimoto T, Koya D, Haneda M, Kashiwagi A, Kikkawa R: Cerebral microvascular disease predicts renal failure in type 2 diabetes. J Am Soc Nephrol 2010;21:520-526.

39 Wada M, Nagasawa H, Kurita K, Koyama S, Arawaka S, Kawanami T, Tajima K, Daimon $\mathrm{M}$, Kato T: Microalbuminuria is a risk factor for cerebral small vessel disease in community-based elderly subjects. J Neurol Sci 2007; 255:27-34.

40 Wada M, Nagasawa H, Iseki C, Takahashi Y, Sato H, Arawaka S, Kawanami T, Kurita K, Daimon M, Kato T: Cerebral small vessel disease and chronic kidney disease (CKD): results of a cross-sectional study in community-based Japanese elderly. J Neurol Sci 2008;272:36-42.

41 Wada M, Nagasawa H, Kawanami T, Kurita K, Daimon M, Kubota I, Kayama T, Kato T: Cystatin $\mathrm{C}$ as an index of cerebral small vessel disease: results of a cross-sectional study in community-based Japanese elderly. Eur J Neurol 2010;17:383-390.
42 Weiner DE, Bartolomei K, Scott T, Price LL, Griffith JL, Rosenberg I, Levey AS, Folstein MF, Sarnak MJ: Albuminuria, cognitive functioning, and white matter hyperintensities in homebound elders. Am J Kidney Dis 2009;53: 438-447.

43 Putaala J, Haapaniemi E, Gordin D, Liebkind R, Groop PH, Kaste M, Tatlisumak T: Factors associated with impaired kidney function and its impact on long-term outcome in young ischemic stroke. Stroke 2011;42:24592464.

44 Bamford J, Sandercock P, Dennis M, Burn J, Warlow C: Classification and natural history of clinically identifiable subtypes of cerebral infarction. Lancet 1991;337:1521-1526.

45 Adams HP Jr, Bendixen BH, Kappelle LJ, Biller J, Love BB, Gordon DL, Marsh EE 3rd: Classification of subtype of acute ischemic stroke. Definitions for use in a multicenter clinical trial. TOAST. Trial of Org 10172 in Acute Stroke Treatment. Stroke 1993;24:35-41.

46 Longstreth WT Jr, Dulberg C, Manolio TA, Lewis MR, Beauchamp NJ Jr, O'Leary D, Carr J, Furberg CD: Incidence, manifestations, and predictors of brain infarcts defined by serial cranial magnetic resonance imaging in the elderly: the Cardiovascular Health Study. Stroke 2002;33:2376-2382.

47 Knopman DS, Mosley TH Jr, Bailey KR, Jack CR Jr, Schwartz GL, Turner ST: Associations of microalbuminuria with brain atrophy and white matter hyperintensities in hypertensive sibships. J Neurol Sci 2008;271:53-60.

48 Pantoni L, Poggesi A, Basile AM, Pracucci G Barkhof F, Chabriat H, Erkinjuntti T, Ferro JM, Hennerici M, O’brien J, Schmidt R, Visser MC, Wahlund LO, Waldemar G, Wallin A, Inzitari D: Leukoaraiosis predicts hidden global functioning impairment in nondisabled older people: the LADIS (Leukoaraiosis and Disability in the Elderly) Study. J Am Geriatr Soc 2006;54:1095-1101.

49 Hashimoto J, Aikawa T, Imai Y: Large artery stiffening as a link between cerebral lacunar infarction and renal albuminuria. Am J Hypertens 2008;21:1304-1309.

50 Potter G, Doubal F, Jackson C, Sudlow C, Dennis M, Wardlaw J: Associations of clinical stroke misclassification ('clinical-imaging dissociation') in acute ischemic stroke. Cerebrovasc Dis 2010;29:395-402.

51 Jackson CA, Hutchison A, Dennis MS, Wardlaw JM, Lewis SC, Sudlow CL: Differences between ischemic stroke subtypes in vascular outcomes support a distinct lacunar ischemic stroke arteriopathy: a prospective, hospitalbased study. Stroke 2009;40:3679-3684.

52 Rowat A, Graham C, Dennis M: Dehydration in hospital-admitted stroke patients: detection, frequency, and association. Stroke 2012; 43:857-859. 